\title{
Actualidad institucional y económica de España en el marco de la Unión Europea (Enero 2019)
}

\author{
Beatriz Iñarritu \\ Profesora de la «Deusto Business School», Universidad de Deusto
}

doi: http://dx.doi.org/10.18543/ced-60-2019pp387-407

Sumario: I. Introducción.-II. El Estado de la Integración.-III. Cuestiones generales de la actualidad económica

\section{Introducción}

Durante el segundo semestre de 2018, la UE ha podido alcanzar ciertos acuerdos importantes en el ámbito de la profundización de la Unión Económica y Monetaria con el compromiso para diseñar a lo largo de 2019 el mecanismo financiero que deberá ser el embrión del presupuesto común para la Eurozona, y a pesar de la falta de acuerdo en relación al Fondo Europeo de Garantía de Depósitos con el que debería culminarse la Unión Bancaria.

Las negociaciones respecto al Brexit parecen haber llevado al Reino Unido a la aceptación de permanencia en la unión aduanera durante un período transitorio que podría prorrogarse más allá del 31 de Diciembre de 2020, aunque la decisión final se trasladaba al Parlamento británico.

También fueron noticia la celebración del 20 aniversario del nacimiento del Euro el 1 de Enero de 1999 y la votación del Parlamento Europeo contra Hungría por sus prácticas antidemocráticas contrarias a los valores europeos, lo mismo que el acuerdo comercial firmado con Japón, importante contrapunto al conflicto con Estados Unidos, y la finalización de los estímulos monetarios extraordinarios puestos en marcha por el Banco Central Europeo en Marzo de 2015.

During the second six months of 2018, the European Union has been able to take certain important steps in the deepening of the Economic and Monetary Union with the compromise of designing in 2019 the financial facility that should be the precedent of the common budget for the Eurozone, and despite the lack of agreement about the European 
Deposit Insurance Scheme, the last project needed to conclude the Banking Union.

The progresses in the Brexit process seem to have led the United Kingdom to accept remaining in the customs union during a transitional period, which could be extended beyond the $31^{\text {st }}$ December 2020, although the final decision was still depending on the British Parliament.

Remarkable were also the news about the $20^{\text {th }}$ anniversary of the Euro's birth on 1 January 1999 and the vote in the European Parliament against Hungary because of its anti-democratic practices contrary to the European values, as well as the trade agreement with Japan, an important counterpoint to the conflict with the United States, and the end of the extraordinary monetary stimulus initiated by the European Central Bank in March 2015.

\section{El estado de la integración}

\section{Acuerdos sobre la profundización en la Unión Económica y Monetaria}

A pesar de la expectación creada por el acuerdo franco-alemán sobre los avances en la Gobernanza Económica del Euro, y en particular sobre el presupuesto de la Eurozona y la culminación de la Unión Bancaria, los Jefes de Estado y de Gobierno de la Unión Monetaria, reunidos en la Cumbre del Euro el 29 de Junio, no alcanzaron compromisos relevantes.

Un numeroso bloque de países, liderado por Holanda (con los nórdicos Dinamarca, Finlandia y Suecia, los bálticos Estonia, Letonia y Lituania, e Irlanda), vetó la propuesta de crear un primer presupuesto común de los Diecinueve. Todos ellos mostraron su frontal rechazo a toda iniciativa que suponga la mutualización de riesgos o cualquier atisbo de Unión Fiscal, y defendieron, en cambio, la receta de la disciplina fiscal en cada uno de los Estados miembros como fórmula clave para afrontar nuevas crisis.

Ningún avance se registró respecto al presupuesto de la Eurozona que pedían París y Berlín, bajo la posible forma de un «minifondo» de estabilización de inversiones de 30.000 millones de euros propuesto por la Comisión Europea.

La cumbre se limitó a encargar un calendario para la negociación del tercer pilar de la Unión Bancaria, el Fondo Común de Garantía de Depósitos, FCGD, o EDIS por sus siglas en inglés, el tercer pilar de la unión bancaria.

Con este FCGD, los ahorros de los europeos hasta 100.000 euros por cuenta corriente o depósito, gozarían del mismo nivel de protección, independientemente del país en el que se encuentre su banco. Se evitarían así 
fugas de capitales como las ocurridas en España durante la crisis de 2012 o en Grecia en 2015, y por esta razón es una cuestión prioritaria para el gobierno español. Sin embargo, Berlín y otros países del norte lo bloquearon porque temen acabar pagando la factura de los agujeros en los bancos del resto de países de la Eurozona; exigen la previa reducción del riesgo bancario y de la cartera de Deuda nacional en las entidades bancarias.

Uno de los pocos puntos en los que se alcanzó un principio de acuerdo entre las capitales fue el refuerzo del Mecanismo Europeo de Estabilidad (MEDE), el fondo de rescate de la UME que tiene una capacidad de crédito de hasta 500.000 millones de euros.

En París y Berlín se habló incluso de rebautizarlo como Fondo Monetario Europeo, pero los líderes europeos no respaldaron finalmente este término a pesar de que sí admitieron la necesidad de que, en en el futuro, el MEDE otorgue con más facilidad líneas de crédito preventivas a aquellos países que sufran una crisis de liquidez, para no tener que recurrir a un rescate completo.

Y los Diecinueve también mostraron su acuerdo para que el MEDE se convierta en el avalista común de último recurso del Fondo Único de Resolución, cuya función es financiar las liquidaciones de bancos, aunque las condiciones o la fecha en que estará disponible esta «red de seguridad» aún no fueron decididas en Junio.

Ya en Diciembre, el día 4, el Eurogrupo logró un acuerdo de mínimos para esta «reforma del Euro». En efecto, los ministros de Finanzas de la zona Euro consiguieron avanzar en los dos temas que habían conseguido un mayor consenso en Junio, el respaldo fiscal para las quiebras bancarias y la reforma del MEDE.

Entre las medidas acordadas sobre esta reforma del MEDE destacan las llamadas «cláusulas de acción colectiva», CAC, que se introducirán en 2022 y que permitirán que una mayoría de acreedores pueda imponer al resto reestructuraciones de Deuda.

Sin embargo, los ministros no consiguieron superar el desacuerdo en relación al presupuesto de la zona Euro, con la perspectiva de que este presupuesto se convierta en una herramienta fiscal de estabilización de las Economías de la Eurozona ante nuevas recesiones. Las discrepancias entre los países, liderados por Francia y Holanda en las posturas más antagónicas, condujeron a duras declaraciones. Mientras el ministro francés, Le Maire, afeó a Holanda que liderase un «club de países» estrictos con la disciplina fiscal que, en su opinión, «no deja avanzar al proyecto europeo», su homólogo holandés, Hoekstra, afirmó que el proyecto francoalemán había pasado de ser un «elefante» a convertirse en un «ratón enjaulado».

Tampoco se lograron avances sobre el Fondo de Garantías de Depósitos. Los ministros del Euro dieron la misma respuesta que en junio, es decir, optaron por aplazar el debate a nuevas reuniones en 2019. 
Estas negociaciones del Eurogrupo fueron el preámbulo de la Cumbre del Euro celebrada el 14 de Diciembre que finalizó con un mensaje esperanzador.

En efecto, los líderes de la UME lograron acordar un calendario para dotar a la Unión Monetaria de un presupuesto, a pesar de que el veto de los países del norte impidió que este embrión presupuestario asuma inicialmente una función anticrisis.

El principal promotor del proyecto, el presidente francés Macron, consiguió el compromiso de sus homólogos para iniciar la puesta en marcha del necesario «brazo fiscal» para la zona Euro cuyo diseño debería estar a punto en junio de 2019, y a pesar de que en la misma declaración de la Cumbre del Euro se establecía el límite de sus funciones al fomento de la «competitividad» y la «convergencia» de los Estados que comparten la moneda única. Esta definición reflejaba el mencionado veto de los países del norte, liderados por Holanda, a que este fondo pueda servir para hacer frente a futuras crisis.

Los líderes de la Eurozona acordaron que durante el primer semestre de 2019 los ministros de Finanzas, en el marco del Eurogrupo, se encargarían de diseñar ese mecanismo, buscar fórmulas para implantarlo y fijar un calendario para su puesta en marcha.

La base para su desarrollo son dos propuestas de la Comisión Europea: un fondo para financiar reformas (20.000 millones) y otro para inversiones en tiempos de crisis (30.000 millones), y estaría enmarcado, previsiblemente en el nuevo Marco Financiero Plurianual 2021-2027.

Pese a tener que renunciar a la función anticrisis, fuentes del gobierno francés declararon que este acuerdo «hubiera sido imposible hace un año» y advirtieron de que, en todo caso, Francia no renunciaba ni a la idea ni a la voluntad de que la zona Euro «pueda tener una capacidad fiscal para hacer frente a los shocks económicos y financieros».

Tampoco la Comisión Europea renunciaba a crear una capacidad fiscal para la zona Euro. En la rueda de prensa posterior a la cumbre, Juncker señaló que «aunque no suscite unanimidad, recuperará su propuesta para un fondo de inversiones que actúe en momentos de crisis». El presidente del Ejecutivo comunitario dijo estar convencido de que un presupuesto con función estabilizadora «acabará encontrando cabida», porque el Euro «no puede vivir sin instrumentos que permitan reaccionar a golpes externos».

Por su parte, el proyecto del Fondo Común de Garantía de Depósitos, la pieza clave para culminar la Unión Bancaria, no registró avance alguno ante la negativa de Berlín a compartir riesgos sin que éstos se hayan reducido.

La desconfianza mutua entre los socios retrasaba sine die la culminación de la Unión Bancaria. «Me da la impresión de que avanzamos, pero no con suficiente rapidez», lamentó Jean-Claude Juncker. 


\section{Parlamento Europeo: votación para sancionar a Hungría por prácticas contrarias al Estado de Derecho}

El pleno del Parlamento Europeo votó en Septiembre la activación del procedimiento sancionador del artículo 7 del Tratado de la UE contra Hungría por incumplir persistentemente las reglas y los valores democráticos que fundamentan la integración comunitaria.

Por 448 votos a favor, 197 en contra y 48 abstenciones, la Eurocámara aprobó un informe elaborado por la eurodiputada holandesa Judith Sargentini, del grupo de los Verdes, que instaba al Consejo de la UE a aplicar a Hungría el mencionado artículo 7, que podría implicar la imposición de una serie de sanciones disciplinarias sin precedentes contra un Estado miembro, como la suspensión del derecho de voto en el seno del propio Consejo de ministros comunitario.

La votación fue calificada como histórica ya que por primera vez el Parlamento Europeo daba luz verde a la activación de este procedimiento.

En el origen de la denuncia sobre Hungría se encuentran diferentes tipos de prácticas rechazadas por las instituciones europeas, como la negativa del gobierno húngaro a acoger refugiados, las trabas a la libertad de prensa y a la independencia judicial, el control sobre la actividad de las ONG, la corrupción o la aprobación de las leyes sobre universidades que obstaculiza la actividad en el país de la Universidad Centroeuropea del magnate George Soros.

Y, por otra parte, los medios también se hicieron eco del hecho de que la votación necesitaba de un consenso parlamentario que requería, necesariamente, del voto favorable de al menos parte del Partido Popular Europeo, PPE, grupo del que también formaba parte el partido del gobierno húngaro. De hecho, y ante la fuerte controversia que planteaba el caso, el PPE decidió finalmente dar libertad de voto a sus miembros, lo que provocó la división entre los populares europeos.

El PPE, que durante años ha mantenido una incómoda convivencia con el primer ministro húngaro Viktor Orbán en aras de lograr una formación lo más amplia posible, tanto en número de escaños en el Parlamento como en número de Gobiernos teóricamente afines, evidenció una inusual división, entre dos bloques, el de las formaciones más tolerantes con el gobierno húngaro, como la española, la italiana y parte de la francesa, frente al bloque de los que abogan por un castigo ejemplar e incluso la expulsión del grupo (eurodiputados nórdicos, del Benelux y de parte de Alemania).

De hecho, no se descartaba que la votación de Septiembre pudiera haber desembocado en una escisión que hubiera arrastrado a una parte importante del grupo, sobre todo de eurodiputados de países del Este, ya que en el mismo debate parlamentario previo a la votación se escenificó la tensión 
entre el mandatario magiar y Manfred Weber, el líder del PPE en la Eurocámara.

El éxito del proceso se anuncia, sin embargo, muy complicado ya que la apertura formal del procedimiento requiere, de acuerdo con el artículo 7, de una mayoría supercualificada de cuatro quintos de los Estados miembros en el Consejo de Ministros de la UE. Y, posteriormente, también sería precisa la votación por unanimidad de los Estados miembros, requisito que se antoja prácticamente imposible de ser cumplido por el rechazo que muestran diferentes Estados, y en particular Polonia.

Esto es así porque, en efecto, el único precedente que existe sobre este procedimiento lo protagonizó Polonia. A finales de 2017 la Comisión Europea también solicitó la aplicación del artículo 7 respecto al ejecutivo polaco, que había aprobado una serie de reformas judiciales que ponían en tela de juicio su independencia, aunque finalmente la acción de Bruselas no tuvo mayor repercusión ya que nunca fue aprobada por el Parlamento Europeo.

\section{Veinte aniversario del Euro y presentación del Plan para reforzar su papel internacional}

La Comisión Europea presentó el 5 de Diciembre un plan para fortalecer el papel del Euro en la escena internacional y desafiar el dominio del dólar como principal divisa global, con una atención especial al ámbito de la energía, ya que en este sector Bruselas desea impulsar el uso de la moneda europea para reducir la «exposición» de la UE al billete verde, lo que «supone incertidumbres, riesgos y costes».

Asimismo, Bruselas contextualizaba el plan en el hecho de que el Euro, a pesar de ser la segunda divisa mundial, se utiliza en el 36\% de las transacciones internacionales y únicamente representa el $18 \%$ de las reservas de divisas en Bancos Centrales extranjeros, mientras que el dólar, que se utiliza en el $40 \%$ de los pagos globales, representa el $62 \%$ de las reservas.

La idea del plan ya fue planteada por el presidente de la Comisión, Jean Claude Juncker, en Septiembre en su discurso sobre el Estado de la Unión ante el Parlamento Europeo. Entonces el luxemburgués mencionó dos ejemplos que calificó de absurdos: la UE es el mayor importador mundial de energía (petróleo y gas, fundamentalmente), su factura anual asciende a 300.000 millones de euros, y un $85 \%$ se paga en dólares estadounidenses a pesar de que solo el 2\% proviene de Estados Unidos; y citó como segundo ejemplo el caso de las aerolíneas europeas, que utilizan también la divisa americana para comprar aviones, aunque los fabrique un consorcio europeo como Airbus.

«El Euro es una moneda joven pero exitosa y tenemos que desarrollar su papel en el mundo para que refleje el peso político, económico y finan- 
ciero de la Eurozona», señaló en Diciembre el vicepresidente del Ejecutivo comunitario para el Euro, Valdis Dombrovskis.

Esta declaración de intenciones planteaba una serie de acciones para aumentar las transacciones en euros.

En concreto, se contemplaban acciones en los dos sectores estratégicos mencionados, la energía y la aeronáutica. En el ámbito de la energía, Bruselas planteaba que los contratos que suscriban los Estados miembros con terceros países se realicen en euros. Rusia, Oriente Medio y África son los principales suministradores, y la UE tiene una posición de fuerza por ser el mayor importador de energía del mundo. El cambio de divisa será más difícil en la compra de petróleo ya que, a pesar de tener también un peso importante en la compra, la Organización de Países Exportadores de Petróleo (OPEP) opera con dólares. En todo caso, la Comisión entiende que sí sería factible pagar el gas natural en euros (el 70\% de las compras de este combustible aún se realiza con el billete americano).

El ejecutivo comunitario pedía, además, que el Euro se utilice en los contratos celebrados en el marco de acuerdos intergubernamentales sobre energía, entre Estados miembros y terceros países, así como en las transacciones realizadas por parte de empresas que prestan servicios financieros.

Y el plan también proponía reforzar la utilización del Euro en el negocio aeronáutico, ya que la práctica totalidad de la facturación de los fabricantes se computa en dólares estadounidenses, incluso dentro de la zona del Euro. De hecho, y según el informe, en torno al 60\% de los ingresos de Airbus está denominado en esa divisa.

El Ejecutivo comunitario anunció su intención de lanzar una serie de consultas públicas, cuyos resultados presentará en verano de 2019. Estas consultas explorarán con más detalle las razones por las que el Euro no se usa en muchas transacciones internacionales relevantes y ayudarán a identificar las condiciones que podrían permitir la promoción del Euro en transacciones con negocios europeos.

Por otra parte, la moneda única cumplió el veinte aniversario de su nacimiento el 1 de Enero de 2019.

En la nochevieja de 1998 once países de la Unión Europea daban la bienvenida al Euro, a pesar de que sus monedas y billetes no se pondrían en circulación hasta tres años más tarde. Ese mismo día, el primero de 1999, los primeros once Estados de la Eurozona renunciaban a su moneda nacional y el Banco Central Europeo iniciaba su labor de autoridad monetaria en esta Unión Monetaria.

Dos décadas después, y tras haber vivido una recesión que comprometió su propia supervivencia, la Eurozona afronta los retos de completar su gobernanza económica y de relanzar su papel en las transacciones internacionales. 
«El Euro se ha convertido en un símbolo de unidad, soberanía y estabilidad. Ha brindado prosperidad y protección a nuestros ciudadanos y debemos asegurarnos de que continúe haciéndolo. Por ello trabajamos con tesón para completar nuestra Unión Económica y Monetaria e impulsar el papel internacional del Euro», señaló Jean Claude Juncker con motivo de la celebración del aniversario.

\section{Brexit: Acuerdo sobre las condiciones del período transitorio del Brexit}

En Septiembre se celebró un Consejo Europeo en la ciudad austríaca de Salzburgo en la que los líderes de los Veintisiete tuvieron la oportunidad de analizar y debatir sobre el plan presentado por la premier británica Theresa May para abandonar la Unión Europea.

May había llegado a la cumbre para plantear una especie de ultimátum para forzar la aprobación de su plan de salida, conocido como «Chequers» en referencia al plan aprobado por el Gobierno británico en el verano de 2018 en esta residencia campestre de la primera ministra, y llegó a pedir a la UE que flexibilizara su posición para tratar de llegar a un acuerdo y que no le pidieran «cosas imposibles».

Sin embargo, el alegato de May sirvió de poco. Los Veintisiete dejaron claro su indiferencia hacia las amenazas de May y cerraron filas con el negociador jefe de la UE, Michel Barnier, partidario de mantener una fuerte presión sobre el Reino Unido.

Donald Tusk, presidente del Consejo Europeo, instó a Londres a rehacer su propuesta de Brexit para poder llegar a un acuerdo que evitara una salida caótica de la Unión Europea el 29 de marzo de 2019.

A pesar de reconocer la evolución positiva del enfoque británico y su voluntad de minimizar los efectos negativos del Brexit, incluida la disposición de Londres para cooperar estrechamente en seguridad y política exterior, Tusk destacó que en otros asuntos, como la cuestión irlandesa o el marco de cooperación económica, «la propuesta de Reino Unido necesitaría ser rehecha y volver a ser negociada».

De esta forma, May abandonó la ciudad austriaca con la obligación de rehacer un plan con el que había pretendido reservar para el Reino Unido ciertos privilegios en la libre circulación de mercancías y un derecho de veto en la futura legislación comunitaria, sobre todo, en la relativa al sector financiero. La propuesta de Londres también insistía en restringir la inmigración europea.

El rechazo al plan fue interpretado por la prensa británica como una «emboscada» y como una «humillación». La propia May llegó a insinuar la responsabilidad de la UE en un eventual descarrilamiento de las negociacio- 
nes que podría conducir a un Brexit caótico el 29 de marzo de 2019, y dejó entrever que Bruselas habría rechazado el pacto para forzar un caos político en Reino Unido.

La cita de Salzburgo, lejos de ofrecer un respiro a May, tuvo el efecto de redoblar la presión sobre su gobierno ante la división existente en el país y que enfrenta a quienes desean una salida radical y a quienes aspiran a dar la vuelta al Brexit y permanecer en la Unión.

En una nueva cumbre de los líderes europeos celebrada en Octubre, la premier británica no presentó ninguna propuesta para evitar una frontera entre el territorio británico de Irlanda y el resto de la isla, el asunto más espinoso de las negociaciones. Y ante esta situación, la UE se mostró dispuesta a alargar un año más el periodo transitorio posterior al Brexit, hasta finales de 2021.

Londres y Bruselas se manifestaron de acuerdo sobre la necesidad de ganar tiempo. Este alargamiento del periodo transitorio significaría la permanencia del Reino Unido en la unión aduanera de la UE y el acatamiento del Derecho comunitario y de las sentencias del Tribunal de Luxemburgo, pero sin voz ni voto, y contribuyendo al presupuesto de la UE con una cifra en torno a los 10.000 millones de euros anuales, condiciones todas ellas rechazadas frontalmente por los brexiters.

Los 27 ofrecieron más tiempo al gobierno de May a cambio de que realizase una contraoferta para resolver el punto más conflictivo, relacionado con la frontera de Irlanda.

Fue el 13 de Noviembre cuando Bruselas y Londres anunciaron que sus equipos negociadores habían llegado a un principio de acuerdo que contemplaba mantener a todo el Reino Unido dentro de la unión aduanera durante el período de transición, que se iniciaría a partir del 29 de Marzo de 2019 y que finalizaría, en principio, el 31 de Diciembre de 2020, situación que podría prolongarse varios meses más, hasta que ambos ejecutivos alcancen un acuerdo sobre su futura relación comercial.

El acuerdo contemplaba, en efecto, la posible extensión del periodo transitorio más allá del fin de 2020 en el supuesto de que se llegue a 2021 sin un acuerdo comercial (lo que incluye la solución sobre la frontera entre Irlanda del Norte e Irlanda) y si Londres lo solicitase antes del 1 de Julio de 2020.

Otro capítulo destacado del acuerdo se refería a los derechos de los ciudadanos. Más de tres millones de nacionales de los Veintisiete residentes en el Reino Unido y más de un millón de británicos residentes en el bloque comunitario no deberían verse afectados por el Brexit, ya que mantendrán, previsiblemente, todos los derechos garantizados por la libre circulación de personas, incluidos los derechos de residencia, trabajo, estudio, reunificación familiar y asistencia sanitaria, y tanto para quienes se encuentren en el 
otro bloque antes del 29 de Marzo de 2019 como para los que lleguen durante el período transitorio.

Y por lo que se refiere a la «factura» del Brexit, el acuerdo fijaba que el Reino Unido seguiría realizando sus aportaciones al presupuesto de la UE, unos 7.000 millones de euros netos anuales, durante los dos años siguientes, 2019 y 2020. La deuda británica con la Unión se estima en un saldo neto favorable a Bruselas de alrededor de 50.000 millones.

Ante los miembros de su gobierno, May señaló que «había cerrado el mejor pacto posible en defensa del interés nacional», lo que no evitó la dimisión de dos de sus ministros. Primero presentó su renuncia el responsable del Brexit, Dominic Raab, quien, paradójicamente, había estado al frente de las negociaciones y que, sin embargo, declaró al dimitir que «los términos propuestos por la primera ministra contienen errores muy relevantes», añadiendo que «si este acuerdo entra en vigor provocará un devastador efecto en la confianza de la ciudadanía en la democracia». Y poco después, dimitía la ministra de Trabajo y Pensiones, Ester McVey, quien señalaba en su renuncia que el acuerdo «no hacía honor al resultado del referéndum».

Apenas unos días después, en un Consejo Europeo extraordinario, celebrado el 25 de Noviembre y que apenas duró 25 minutos, los Veintisiete líderes europeos dieron su apoyo al pacto de salida y a la declaración política que perfilaba la futura relación entre los Veintisiete y Londres.

Por su parte, la votación en el pleno del Parlamento británico, inicialmente prevista para el 11 de Diciembre, fue finalmente retrasada hasta al 15 de Enero de 2019, ante el previsible rechazo que el acuerdo provocaría entre las filas laboristas y entre un buen número de diputados tories, conservadores del mismo partido de May.

En efecto, en un debate parlamentario que siguió a la presentación del acuerdo, el líder del partido laborista, Jeremy Corbyn, acusó a la premier de dirigir un «gobierno en el caos» y de presentar «un acuerdo medio cocinado que deja al Reino Unido sin voz ni voto», por lo que anunciaba el voto en contra de su formación. Y por parte de los correligionarios de partido de la premier se escucharon numerosas críticas y peticiones de rectificación.

En todo caso, para ambas partes, el propio Reino Unido y los Veintisiete, el escenario del «no acuerdo» sería el más disruptivo de todos, ya que supondría una ruptura brusca que alteraría el flujo comercial (incluyendo la restauración de barreras arancelarias, regulatorias y políticas), agitaría los mercados y rompería la cooperación en materia de seguridad. El caos podría afectar a multitud de sectores, como el transporte aéreo, los medicamentos, los alimentos y, por supuesto, al estatus legal de los expatriados en cada territorio. 


\section{Cuestiones generales de la actualidad económica}

1. BCE: Finalización del Programa de compras masivas de deuda (QE) en Diciembre de 2018

Tras el anuncio realizado en Junio, el Banco Central Europeo, BCE, dio por finalizado en Diciembre el programa excepcional de compras masivas de activos, bonos soberanos y corporativos, conocido como «Quantitative Easing', QE.

Este programa, que se inició en Marzo de 2015, fue interpretado entonces por una mayoría de expertos y medios económicos como un intento prácticamente desesperado para apoyar a la maltrecha Economía de la Eurozona y alejar el fantasma de la deflación. Tras inyectar 2,6 billones de euros, más del doble del PIB español, el BCE afirmaba en Diciembre que su objetivo había sido cumplido.

En cualquier caso, y para garantizar una cierta gradualidad en la retirada de estímulos, el BCE anunció en el comunicado posterior a la celebración de su Consejo de Gobierno de Diciembre que continuaría reinvirtiendo la Deuda conforme ésta fuera venciendo «durante un periodo prolongado», plazo que podría alargarse más allá de la primera subida de tipos, «y, en todo caso, durante el tiempo que sea necesario para mantener unas condiciones de liquidez favorables», añadía el texto.

Mario Draghi afirmó, en la rueda de prensa que siguió a la reunión que «el programa no termina, sino que continúa porque nos hemos comprometido a mantener un stock de Deuda por un periodo prolongado».

Respecto a los tipos de interés, el italiano afirmó que no habría subidas al menos hasta el verano de 2019 o hasta una fecha posterior, dependiendo de si el BCE lo considerase necesario para acercarse al objetivo de una inflación por debajo pero cerca del $2 \%$.

A comienzos de Enero, fuentes del Banco comunicaron, en efecto, su intención de adquirir en torno a 202.772 millones de euros en bonos a lo largo de 2019, para reponer la Deuda de su cartera que alcance su vencimiento.

A lo largo de los casi cuatro años de programa de compras, la adquisición de bonos se ha repartido entre los distintos países de la zona Euro atendiendo a la clave de sus aportaciones al capital del BCE, una cifra que viene determinada por el peso del PIB y la población de cada país, y que se revisa cada cinco años.

Dado que las reinversiones se realizarán, previsiblemente, en las mismas regiones donde los bonos vayan alcanzando su vencimiento, esta circunstancia podría beneficiar a los países que han perdido peso en la clave del capital del BCE tras la última revisión realizada en 2018 y que entró 
en vigor el 1 de Enero de 2019. Este es el caso de España, que ha pasado del $8,8 \%$ al $8,3 \%$ en esta nueva clave del capital.

2. Semestre Europeo 2019: Estudio Prospectivo Anual para el Crecimiento (EPAC) e Informe sobre el Mecanismo de Alerta (IMA)

La Comisión Europea presentó en Noviembre el llamado «Paquete de Otoño» que incluye los dos análisis económicos con los que se iniciaba el Semestre Europeo 2019: el Estudio Prospectivo Anual para el Crecimiento, EPAC, y el Informe sobre el Mecanismo de Alerta, IMA.

En un comunicado de prensa Bruselas señalaba que «el ciclo de coordinación de políticas económicas y sociales del Semestre Europeo de 2019 se acomete en un contexto de crecimiento sostenido, aunque menos dinámico, y marcado por un clima de gran incertidumbre».

El EPAC del Semestre, que establece las prioridades económicas y sociales generales para el próximo año, requería que la UE y sus Estados miembros adopten medidas políticas «decisivas y concertadas» para lograr un crecimiento integrador y sostenible.

A escala nacional, el informe se centraba en los esfuerzos políticos enfocados a realizar inversiones de calidad y en las reformas que potencien el crecimiento de la productividad, la integración y la capacidad institucional, y que garanticen, al mismo tiempo, la estabilidad macrofinanciera y unas finanzas públicas saneadas. A escala de la UE, el EPAC señalaba como prioridades la profundización del mercado único, la culminación de la arquitectura de la Unión Económica y Monetaria y el impulso de los principios establecidos en el pilar europeo de Derechos Sociales.

Por su parte, el IMA, que sirve de base para la detección de desequilibrios macroeconómicos en función de un cuadro de 14 indicadores relevantes, identificó trece Estados miembros que debían ser sometidos a un examen exhaustivo en 2019, para determinar si efectivamente están experimentando desequilibrios macroeconómicos.

Se trata de Alemania, Bulgaria, Chipre, Croacia, España, Francia, Irlanda, Italia, Países Bajos, Portugal, Suecia, Grecia y Rumanía.

De esta forma, la Comisión actualizaba la infografía que resume la situación de los países europeos respecto a dos de los procedimientos más relevantes de la Gobernanza Económica de la UE, el Pacto de Estabilidad y Crecimiento, PEC, y el Procedimiento de Desequilibrios Macroeconómicos. 


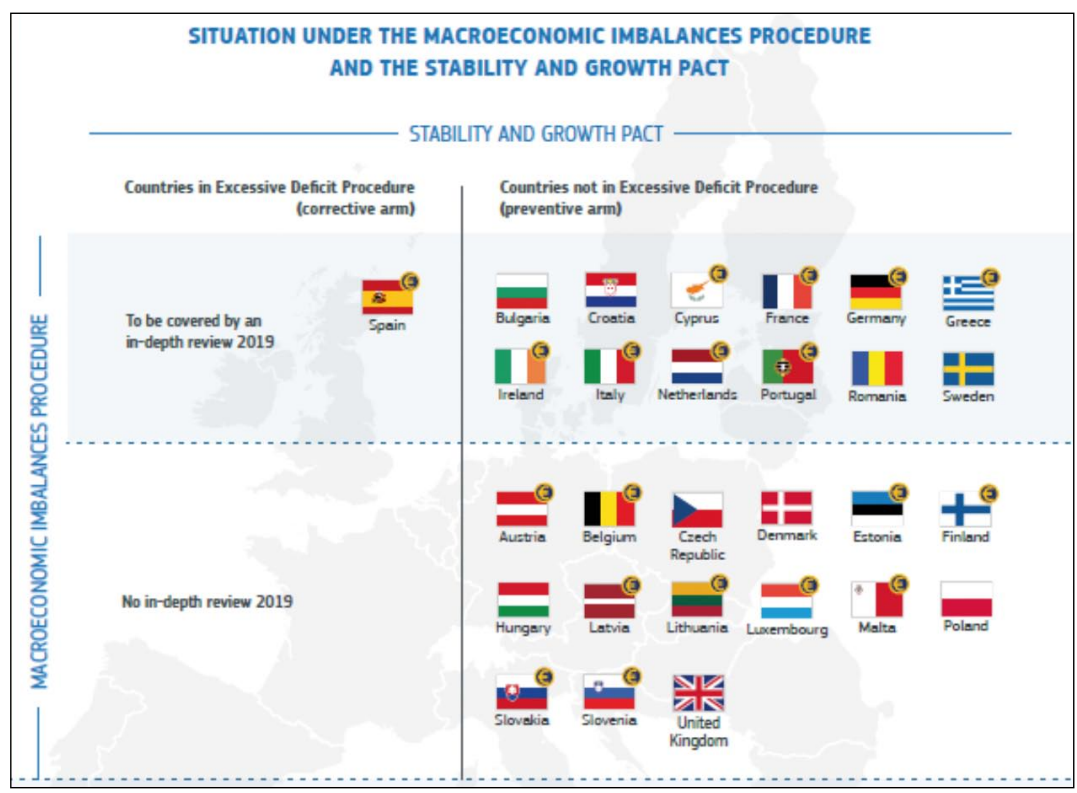

SEMESTRE EUROPEO 2018 (actualización Noviembre 2018)

Fuente: Comisión Europea.

Por otra parte, la Comisión también hizo públicos, en Noviembre, sus dictámenes sobre los proyectos de plan presupuestario, PPP, para 2019 de los diferentes Estados miembros de la zona del Euro, en relación al eventual cumplimiento de las normas del PEC:

- En los casos de diez Estados miembros (Alemania, Austria, Chipre, Finlandia, Grecia, Irlanda, Lituania, Luxemburgo, Malta y los Países Bajos), Bruselas consideró que sus PPP eran conformes con las exigencias del PEC para 2019,

- Respecto a otros tres Estados miembros (Eslovaquia, Estonia y Letonia), la Comisión afirmó que sus PPP se ajustaban «en gran medida» a las disposiciones del PEC para 2019 señalando, sin embargo, que «podrían dar lugar a cierta desviación respecto de sus respectivos objetivos presupuestarios a medio plazo, OMP, o respecto a la senda de ajuste hacia dichos objetivos»,

- En cambio, los PPP de cuatro Estados miembros (Bélgica, Eslovenia, Francia y Portugal) presentaban, según Bruselas, un riesgo de incumplimiento del PEC para 2019, lo que podría traducirse en «una desviación significativa respecto de la senda 
de ajuste hacia el correspondiente objetivo presupuestario a medio plazo».

La Comisión destacaba el hecho de que Grecia se incorporaba por vez primera al Semestre Europeo, una vez que, en efecto, este país había cerrado el 20 de Agosto el tercer programa de asistencia financiera y había recuperado el acceso a los mercados financieros. Bruselas adoptaba en Noviembre un primer informe para este país en el marco del procedimiento de «supervisión reforzada» que se puso en marcha al concluir el rescate del Mecanismo Europeo de Estabilidad, MEDE en Agosto.

A pesar de que la Comisión afirmaba que el PPP para 2019 presentado por Grecia garantizaba el cumplimiento de su compromiso de alcanzar un superávit primario del 3,5\% del PIB (compromiso que debe extenderse hasta 2022), también señalaba que Atenas debía acelerar la aplicación de las reformas y medidas relacionadas con la Deuda, tal como se acordó en la reunión del Eurogrupo de Junio.

Y, por otra parte, Bruselas destacaba en su análisis de los PPP los casos de dos países, Italia y España:

- Respecto a Italia, y tras revisar el PPP revisado presentado por el país alpino el 13 de Noviembre, el ejecutivo comunitario se hacía eco del «grave incumplimiento» de la Recomendación que había sido dirigida a este Estado miembro por el Consejo el 13 de Julio de 2018.

Bruselas afirmaba haber realizado una nueva evaluación del incumplimiento del criterio de la Deuda dado que los planes presupuestarios de Italia para 2019 suponían «una modificación significativa de los factores pertinentes» analizados por la propia Comisión en el mes de Mayo. Y este nuevo análisis sugería que Italia incumplía el criterio de la Deuda incluido en el PEC, por lo que estaría justificado el inicio de un procedimiento por déficit excesivo en este país.

- Y por lo que se refería a España, único país entonces inmerso en el componente correctivo del PEC, la Comisión preveía que el déficit público se situaría por debajo del umbral del $3 \%$ del PIB en 2018 por lo que, en efecto, el país abandonaría el procedimiento por déficit excesivo y pasaría al componente preventivo del PEC a partir de 2019.

Sin embargo, considerando esta nueva situación y el PPP para 2019 presentado por España, la Comisión afirmó que nuestro país corría el riego de incumplir las exigencias del PEC para 2019 ya que, basándose en sus previsiones económicas de otoño de 2018, adivinaba una eventual «desviación significativa respecto de la senda de ajuste exigida hacia el objetivo presupuestario a medio 
plazo» y el incumplimiento del valor de referencia transitorio para la reducción de la Deuda en 2019.

El rechazo del borrador presupuestario italiano elevó la tensión entre Roma y Bruselas, aunque finalmente, y tras varias semanas de negociación, ambos ejecutivos llegaron a un acuerdo el 19 de Diciembre una vez que el gobierno italiano accediera a rebajar el objetivo de déficit público para 2019 desde el 2,4\% del PIB inicial al 2,04\%, compromiso que facilitaba la no apertura de un procedimiento de déficit excesivo.

El primer ministro italiano, Giuseppe Conte, defendió el nuevo plan presupuestario afirmando que «no traicionaba» la confianza de la sociedad italiana porque «mantenía las reformas con «mayor impacto social», haciendo referencia a sus planes de crear una renta ciudadana y de reformar las pensiones.

Con respecto al plan español, el Eurogrupo celebrado el 4 de Diciembre se hizo eco del análisis de la Comisión, y confirmó la advertencia de que tanto el PPP de España como los de Bélgica, Francia, Portugal y Eslovenia podrían incumplir las normas fiscales del PEC.

Los ministros de finanzas de la Eurozona pidieron a estos países que adoptaran medidas adicionales, necesarias para «atajar los riesgos» y garantizar el respeto de las reglas del Pacto.

\section{Comercio: firma del acuerdo con Japón y conflicto comercial con Estados Unidos}

El 17 de Julio tuvo lugar en Tokio la firma del mayor acuerdo de libre comercio del mundo entre la UE y Japón, puesto que entre ambos bloques representan un tercio del PIB mundial.

El Tratado significará importantes beneficios económicos para ambas partes ya que prevé la eliminación prácticamente total de los aranceles en los intercambios bilaterales de bienes y la apertura de mercados para productos y servicios y licitaciones públicas.

En la rueda de prensa que siguió a la firma, el presidente del Consejo Europeo, Donald Tusk, afirmó que «éste es un acto de importancia estratégica enorme para el orden basado en las normas, en un momento en el que algunos cuestionan este orden», lo que se interpretó como una referencia directa a las derivas proteccionistas de Estados Unidos.

Tusk señaló, además, que el acuerdo con Japón no solo «cimentaba» la relación bilateral UE-Japón sino que representaba «un rayo de luz en la oscuridad creciente de la política internacional».

Para cerrar el acuerdo entre Bruselas y Tokio, que se empezó a negociar en 2013, ambas partes han debido realizar importantes concesiones. Por el lado de la Unión, éstas se han centrado, sobre todo, en la eliminación de los 
aranceles a la importación de vehículos nipones (actualmente se sitúan en el $10 \%$ ), aunque se ha establecido un periodo transitorio de siete años para amortiguar el golpe a los fabricantes europeos. Y del lado japonés, se mejora el acceso de los productos agrícolas y de alimentos europeos a este mercado asiático, ya que se reducirán los aranceles de muchos de ellos, y desaparecerán, entre otros, los que se aplican al vino europeo (en torno al 15\%).

Cuando el Tratado entre en vigor, previsiblemente a lo largo de 2019, las empresas europeas podrían ahorrar en torno a 1.000 millones de euros al año según las estimaciones de la Comisión Europea, lo que podría significar un incremento del 13\% en las exportaciones a Japón.

En todo caso, la firma de este importante acuerdo fue considerada por muchos medios como una especie de «aviso» a la administración norteamericana, habida cuenta de los conflictos comerciales que este país ha iniciado con una gran mayoría de sus socios comerciales.

De hecho, apenas unos días después, el 25 de Julio, el presidente de la Comisión Europea, Jean-Claude Juncker, se reunió con Donald Trump en Washington con el objetivo de «reparar» el vínculo comercial entre ambos bloques y después de que el presidente estadounidense hubiera calificado a la UE como su principal «enemigo».

Estados Unidos había decidido imponer, a partir del 1 de Junio, unos nuevos aranceles al acero (25\%) y al aluminio (10\%) procedentes de la Unión, medida que obtuvo la respuesta inmediata de la UE en forma de presentación de una denuncia ante la Organización Mundial del Comercio, OMC, y de la aplicación de nuevos aranceles a unos 200 productos estadounidenses para su venta en el mercado comunitario a partir del 22 de Junio.

En Washington, Juncker y Trump, escenificaron la distensión e, incluso, anunciaron ciertos compromisos encaminados a conseguir la «paz comercial». La UE compraría más soja y gas líquido a su aliado y ambas partes trabajarían conjuntamente para eliminar los aranceles en los bienes industriales. Los derechos arancelarios impuestos al acero y aluminio europeos se reexaminarían, igual que las represalias europeas.

\section{Acuerdo para limitar la comercialización de artículos de plástico de un solo uso}

En Diciembre, las instituciones europeas alcanzaron un acuerdo para vetar la comercialización de una serie de artículos de plástico de un solo uso, decisión que será clave para afrontar el importante reto medioambiental derivado de la plaga de residuos de plástico que inunda las playas y los mares europeos.

El limitado reciclaje de los productos de plástico se ha convertido en un grave problema ya que, según los datos de la Comisión Europea, solo el 30\% 
de los residuos de plástico se reutiliza en la UE. Y, ciertamente, muchos de los productos que no se reciclan acaban en las costas. Un estudio realizado en 2016 por la propia Comisión concluía que el $84 \%$ de las basuras que se acumulan en las playas europeas procede de este tipo de residuos plásticos.

Bruselas había presentado su propuesta de Directiva en mayo, y el 19 de Diciembre se alcanzó un acuerdo con el Parlamento y con los representantes de los Veintiocho.

Tras el preacuerdo político posterior de los ministros de los Estados miembros, se prevé que la Eurocámara también ratifique el acuerdo en el primer trimestre de 2019.

Los productos que se eliminarán de los supermercados europeos en 2021 son de usar y tirar y, según fuentes del ejecutivo comunitario, cuentan con «alternativas fácilmente disponibles y asequibles». Se trata de una lista de 10 productos de plástico, en la que se incluyen los bastoncillos de algodón, los cubiertos y platos, las pajitas, los agitadores de bebidas, los bastones para globos y los recipientes de alimentos hechos con poliestireno expandido. Para otros productos de plástico, como los recipientes de comida, los vasos y las tapas, las acciones previstas se centrarán en la limitación de su uso.

El acuerdo también incluía otra serie de medidas, como que las botellas de plástico contengan un $25 \%$ de material reciclado en 2025 y un $30 \%$ en 2030. Y también, que el $90 \%$ de estas botellas entren en el sistema de reciclaje para 2029.

\section{La Comisión Europea se pronuncia contra el Índice IRPH aplicado en el mercado hipotecario español}

En un informe remitido al Tribunal de Justicia de Luxemburgo, la Comisión Europea defendía, en Septiembre, que las cláusulas hipotecarias referidas al índice IRPH deberían ser nulas si estos créditos no se comercializaron con la debida transparencia, tal como establece la normativa comunitaria de defensa de los consumidores.

En dicho documento el ejecutivo comunitario señalaba que «el profesional está obligado a explicar al consumidor, antes de la firma del contrato, cómo se configura el tipo de referencia, cuál ha sido su evolución en el pasado así como su posible evolución futura, comparándolo con otros tipos utilizados en el mercado».

Debe tenerse en cuenta que este índice hipotecario fue ofrecido a un gran número de clientes bajo su apariencia de mayor estabilidad, aunque se ocultaba el hecho de que desde el año 2001 y hasta la actualidad, el IRPH siempre se ha situado significativamente por encima del euríbor. 
La cuestión es particularmente relevante en la medida en que, en 2017, el Tribunal Supremo español dio por bueno el índice IRPH alegando que es un índice oficial aceptado por los reguladores, tesis que desactivaba un año después la Comisión Europea, al considerar en su informe que el carácter oficial del índice no constituye en sí mismo garantía suficiente para los consumidores españoles, por lo que no debe presuponerse que se haya aplicado con la suficiente transparencia.

El fallo del Supremo supuso, además, una importante limitación en la potestad que las Directivas europeas otorgan a los tribunales nacionales para evaluar si la comercialización de un producto financiero se ha llevado a cabo cumpliendo los requisitos necesarios de diligencia, transparencia e información por parte de la entidad.

Y ha sido, en efecto, el cuestionamiento de la decisión del Supremo por parte de diferentes tribunales de instancias inferiores, el que les ha llevado a presentar diversas cuestiones prejudiciales ante el Tribunal comunitario. Así, el informe elaborado por los servicios jurídicos de la Comisión en Septiembre pretende orientar al Tribunal de la UE en la resolución de una cuestión prejudicial elevada por un Juzgado de Primera Instancia de Barcelona que cuestiona la decisión del Supremo de diciembre del 2017 en relación a una demanda contra Kutxabank sobre una hipoteca referenciada al IRPH.

El Tribunal comunitario deberá decidir si la sentencia del Supremo es compatible con la normativa comunitaria y, por tanto, determinará en qué sentido se han de resolver todas las demandas que se encuentran actualmente paralizadas a la espera, precisamente, de esta decisión de la justicia comunitaria.

$\mathrm{Si}$, como esperan muchos expertos, la sentencia es contraria a la doctrina del Tribunal Supremo, su efecto podría tener un impacto considerable sobre los bancos españoles, de dimensiones similares al efecto de la sentencia sobre las cláusulas suelo.

Según fuentes del sector, en el mercado hipotecario español habría más de un millón de créditos referenciados al IRPH y que han sido mayoritariamente concedidos por las antiguas cajas de ahorro. La sustitución de este índice por el euríbor, y una eventual compensación por el sobrecoste soportado por los consumidores durante años podría significar una nueva perdida millonaria para las entidades financieras españolas.

\section{Competencia : Multa a Google}

La Comisión hizo pública en Julio su decisión de imponer una multa de 4.343 millones de euros a Google, por abuso de posición dominante a través de Android, su sistema operativo para dispositivos móviles, convirtién- 
dose en la sanción más elevada que Bruselas ha impuesto por prácticas monopolísticas a una empresa hasta la fecha.

El ejecutivo comunitario aseguró en un comunicado que el gigante de internet había impuesto «restricciones ilegales» a los fabricantes de dispositivos y operadores móviles para consolidar su posición de dominio en las búsquedas en internet».

En concreto, Bruselas justificaba la multa a Google por obligar a los fabricantes de móviles a preinstalar su buscador y su navegador Chrome como condición para otorgarles la licencia de uso de su tienda de aplicaciones (Play Store). Y también por exigirles el compromiso de no modificar Android (prohibición de venta de dispositivos con la versión abierta de Android) como condición para utilizar las aplicaciones creadas por Google, exigencia que, según Bruselas, impidió crear variantes de Android como la impulsada por Amazon. Una tercera acusación se refería a los pagos realizados a fabricantes y a operadores de redes móviles para que no incluyeran en su oferta preinstalada ningún buscador de la competencia de Google Search.

Pese al elevado importe de la multa, el mayor temor para la multinacional podrían ser las restricciones impuestas por Bruselas para poner coto a sus «prácticas anticompetitivas» con Android, con lo que el imperio móvil de Google podría tambalearse ya que a través de ese sistema operativo ha colocado su buscador, sus servicios Youtube y Google Maps, y su tienda de aplicaciones en más de 2.000 millones de teléfonos móviles en todo el mundo.

De hecho, la Comisión le daba un plazo de 90 días para corregirlas, bajo amenaza de imponerle una multa adicional de hasta el 5\% de la facturación diaria de Alphabet, la matriz de Google.

Frente a estas acusaciones de haber utilizado Android para promocionar sus servicios digitales, la firma estadounidense argumentaba que su plataforma móvil habría permitido a los fabricantes de terminales no tener que comprar o construir un sistema operativo. Y fuentes de la empresa también destacaron que Android es un sistema mucho más abierto que el de Apple, lo que habría impulsado la economía de las apps.

Inmediatamente después del anuncio de la multa, Google aseguró a través de una nota que la recurriría, ya que según afirmaba, «Android ha creado más libertad de elección para todos, no menos». Un portavoz de la multinacional afirmó también que «un ecosistema vibrante, una innovación rápida y unos precios más bajos son los sellos clásicos de una competencia robusta». 


\section{Sobre la autora}

Beatriz Iñarritu Ibarreche es Doctora por la Universidad de Deusto y Licenciada en Ciencias Económicas y Empresariales por la Universidad Comercial de Deusto. Su tesis, «Sistemas de Gobernanza Económica Europea: propuesta de Análisis y Medición», la realizó en el marco del programa de Estudios Internacionales e Interculturales de la Universidad de Deusto. Desde 1988, ha impartido docencia sobre Economía de la UE, en las Licenciaturas de Ciencias Económicas y Empresariales y Derecho de la UD, en la Escuela Universitaria de la Cámara de Comercio de Bilbao, y en los Institutos de Ocio y de Estudios Europeos de la UD. Actualmente, imparte docencia en el Departamento de Economía de Deusto Business School de la UD. Socia de Estrategias Empresariales Europeas, E-3, en la que dirige y desarrolla diferentes proyectos de consultoría en el campo de la internacionalización empresarial y de la UE. Ha sido profesora en el Diploma sobre Derecho Comunitario de la Universidad Iberoamericana de México en colaboración con la UD, impartiendo el módulo sobre «Mercado Interior Europeo e implantación del Euro» en México DF. Es ponente habitual en jornadas organizadas por entidades y organismos empresariales que contribuyen al mayor conocimiento de las diferentes cuestiones relacionadas con la UE y la internacionalización empresarial. Ha participado en un Equipo de Investigación de la UD en el proyecto «Instrumentos Innovadores de Gobernanza de la Unión Europea. Impacto en la gestión pública y en la competitividad regional».

Es autora de la sección «Crónica Comunitaria: la actualidad institucional y económica de España en el marco de la UE» en «Cuadernos Europeos de Deusto», revista editada por la UD. Es coautora de las publicaciones anuales «Guía de la Movilidad en la UE» y «Apuntes sobre la UE y Bizkaia» editadas por la Diputación Foral de Bizkaia. Ha sido coautora del Caso de Internacionalización publicado por el ICEX: «Inauxa, una Pyme en el Mercado Mundial de Automoción».

\section{About the Author}

Beatriz Iñarritu Ibarreche is a Doctor of the University of Deusto and holds a degree in Economics and Business Studies from the Commercial University of Deusto. Her thesis, «Systems of European Economic Governance: Proposal for Analysis and Measurement», was carried out within the framework of the program of International and Intercultural Studies of the University of Deusto. Since 1988, she has lectured on EU Economics, in the Bachelor's of Economics and Business Studies and Law 
at the UD, at the University School of the Chamber of Commerce of Bilbao, and at the Institutes of Leisure and European Studies of the UD. Currently she teaches in the Department of Economics of Deusto Business School of the UD. Partner of European Business Strategies, E-3, in which she directs and develops different consulting projects in the field of business and EU internationalization. She has taught in the Diploma on Community Law of the Universidad Iberoamericana de México in collaboration with the UD, giving the module on «European Internal Market and implementation of the Euro» in Mexico DF. She is a regular speaker at conferences organized by business entities and organizations that contribute to a better understanding of the different issues related to the EU and business internationalization. She has participated in a Research Team of the UD in the project «Innovative Instruments of Governance of the European Union. Impact on public management and regional competitiveness».

She is the author of the section «Community Chronicle: the institutional and economic news of Spain within the framework of the EU» in «Cuadernos Europeos de Deusto», a magazine edited by the UD. She is co-author of the annual publications «Guide to Mobility in the EU» and «Notes on the EU and Bizkaia» edited by the Provincial Council of Bizkaia. She has co-authored the Internationalization Case published by the ICEX: «Inauxa, a SME in the World Automotive Market». 


\section{Derechos de autor}

Los derechos de autor (para la distribución, comunicación pública, reproducción e inclusión en bases de datos de indexación y repositorios institucionales) de esta publicación (Cuadernos Europeos de Deusto, CED) pertenecen a la editorial Universidad de Deusto. El acceso al contenido digital de cualquier número de Cuadernos Europeos de Deusto es gratuito inmediatamente después de su publicación. Los trabajos podrán leerse, descargarse, copiar y difundir en cualquier medio sin fines comerciales y según lo previsto por la ley; sin la previa autorización de la Editorial (Universidad de Deusto) o el autor. Así mismo, los trabajos editados en CED pueden ser publicados con posterioridad en otros medios o revistas, siempre que el autor indique con claridad y en la primera nota a pie de página que el trabajo se publicó por primera vez en CED, con indicación del número, año, páginas y DOI (si procede). Cualquier otro uso de su contenido en cualquier medio o formato, ahora conocido o desarrollado en el futuro, requiere el permiso previo por escrito del titular de los derechos de autor.

\section{Copyright}

Copyright (for distribution, public communication, reproduction and inclusion in indexation databases and institutional repositories) of this publication (Cuadernos Europeos de Deusto, CED) belongs to the publisher University of Deusto. Access to the digital content of any Issue of Cuadernos Europeos de Deusto is free upon its publication. The content can be read, downloaded, copied, and distributed freely in any medium only for non-commercial purposes and in accordance with any applicable copyright legislation, without prior permission from the copyright holder (University of Deusto) or the author. Thus, the content of CED can be subsequently published in other media or journals, as long as the author clearly indicates in the first footnote that the work was published in CED for the first time, indicating the Issue number, year, pages, and DOI (if applicable). Any other use of its content in any medium or format, now known or developed in the future, requires prior written permission of the copyright holder. 